\title{
Towards Sub-micrometer High Aspect Ratio X-ray Gratings by Atomic Layer Deposition of Iridium
}

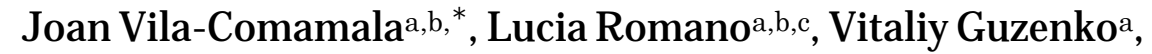 \\ MatiasKagias ${ }^{a, b}$, Marco Stampanoni ${ }^{a, b}$, KonstantinsJ efimovsa,b \\ anstitute for Biomedical Engineering, University and ETH Zürich, 8092 Zürich, \\ Switzerland \\ ${ }^{b}$ Paul Scherrer Institut, 5232 Villigen PSI, Switzerland \\ ${ }^{c}$ Department of Physics and CNR-IMM, University of Catania, 95023 Catania, Italy
}

\begin{abstract}
$\mathrm{X}$-ray grating interferometry is an excellent technique for X-ray phase contrastimagingandX-raywavefrontsensingwith applicationsinmaterialsscience, biology and medical diagnosis. Among other requirements, the method dependsontheavailability of highlyX-rayabsorbingmetallicgratings. Here, wereport on thefabrication and characterization of high aspectratioiridium gratings with a period of one micrometer and a depth of $30 \mu \mathrm{m}$ combining deep reactive ion etching of silicon and atomic layer deposition of iridium. The implementation of such structures can greatly enhance thesensitivity of grating-based X-ray phase contrast imaging and thus, expand further its broad range of applications.
\end{abstract}

Keywords: Grating-based X-ray Interferometry; Deep Reactive Ion Etching of Silicon; Atomic Layer Deposition of Iridium

\footnotetext{
${ }^{*}$ Corresponding author

Email address: joan.vila-comamala@psi.ch (Joan Vila-Comamala)
} 


\section{Introduction}

$\mathrm{X}$-ray grating interferometry $[1,2,3]$ is a prominent method for X-ray wavefront sensing $[4,5]$ and X-ray phase contrastimaging with applications in materials science, biology and medical diagnosis $[6,7,8]$. The technique crucially depends on theavailability of highly X-ray absorbingmetallic gratings. For absorbing typical X-ray photon energies of $20 \mathrm{keV}$ and above, thicknesses of at least 25-30 $\mu \mathrm{m}$ of a high atomic number element, such as gold, are required. On the other hand, typical X-ray grating periods range from 15down toafewmicrometers, and thus, high aspectratiostructuresare necessary for most grating-based X-ray phase contrast imaging setups. Recently,X-ray phaseconstratimagingapplicationsusingeven smallergrating periods have been proposed[7, 9] but currently used fabrication methods can not routinely produce gratings with periods of one micrometer and below. Todate, X-rayabsorption gratingsarecommonlyfabricated combiningoptical orX-ray lithography with gold electroplating in silicon templates[10, 11] or polymer resist molds[12]. However, such fabrication techniques are not easily transferred for the production of sub-micrometer high aspect ratio structures. Here, we propose and demonstrate the production of high aspect ratio ( $>50$ ) iridium gratings with a period of one micrometer and a depth of $30 \mu \mathrm{m}$ combining deep reactive ion etching (RIE) of silicon and atomic layer deposition (ALD) of iridium[13]. Until now, ALD processes have been successfully applied to conformally deposit oxide layers on high aspect (>180) nanostructures[14,15] andALD metal coatingshavealsobeen proven very effective to fabricate high resolution diffractiveX-ray optics in the sub-100 nm structure size range[16, 17]. In this work, we performed 
a thorough optimization of the ALD recipe to extend the conformal metal coating to trench depths of tens of micrometers while keeping the onemicrometer grating periodicity. After scanning electron microscopy inspection, the fabricated gratings were successfully implemented and characterized in a laboratory X-ray phase contrast imaging setup.

\section{Materials and Experimental Methods}

\subsection{Grating-based Phase Contrast $X$-ray Imaging}

Theinteraction ofX-rays with an object can be described byintroducing a complex refractive index, $n=1-\delta+i \beta$, in which $\beta$ and $\delta$ respectively account for theabsorption and phaseshift experimented byX-ray wavefield dueto the specimen[18]. For biological soft tissues with small density differences, the phase shift refractive index variation $\delta$ can be up to three orders of magnitude larger than its absorption counterpart $\beta$, thus allowingX-ray phase contrast imaging to deliver images with much higher contrast in com-

parison to those obtained by conventional X-ray absorption imaging. Among different existing $\mathrm{X}$-ray phase contrast imaging techniques, X-ray grating interferometry obtains excellent contrast and quantitativeinformation of the sampleunder investigation[19, 20]. Grating-based phase contrast imaging can be both performed in synchrotron radiation facilities[2] and usingincoherent laboratory X-ray sources[3]. In the latter case, the setup typically requires the use of two highly absorbing $\mathrm{X}$-ray gratings, $\mathrm{G}_{0}$ and $\mathrm{G}_{2}$, and phase-shifting grating, $\mathrm{G}_{1}$, as schematically represented in Fig. 1. Thecombination of $\mathrm{G}_{0}$ and $\mathrm{G}_{1}$ gratings creates an X-ray intensity modulation at a given downstream distance (Talbot distance) from the $\mathrm{G}_{1}$ phase-shifting 
grating. The $\mathrm{G}_{2}$ gratingis necessary to detect theintensity modulation when the pixel size of the X-ray detector is larger than the period of this X-ray intensity modulation. Afterinserting the samplenear the $\mathrm{G}_{1}$ phase-shifting grating, theX-ray intensity modulation is modified. This change allows the detection of the sample differential phase contrast image by step-scanning one of the three gratings in small steps covering a range of one or several gratingperiods whilerecordingconsecutively thetransmissionX-rayintensity images[2]. The exact geometry and grating periods requirements for an X-ray grating interferometer setup can be calculated using the formulas reported elsewhere[21].

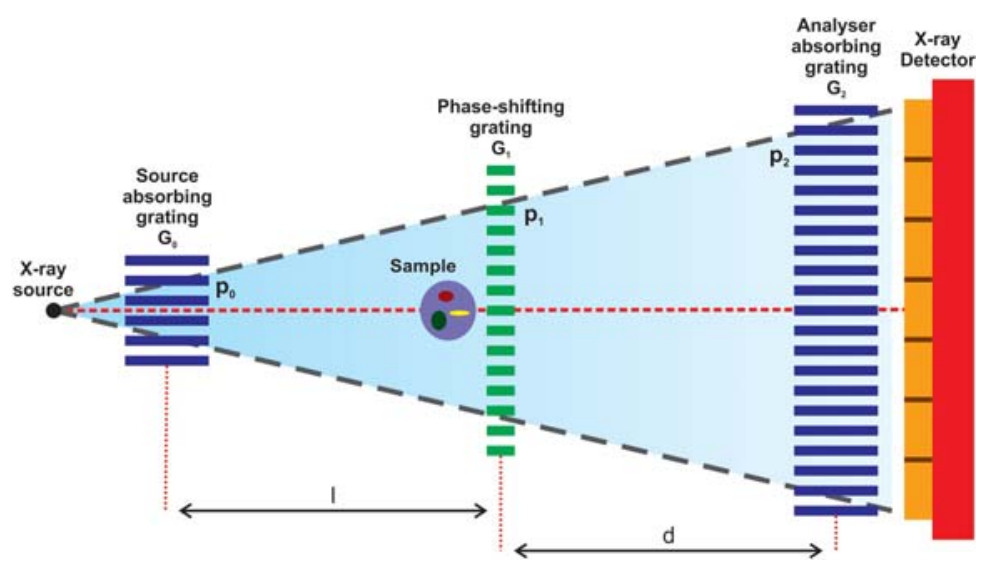

Figure 1: Schematics of laboratory grating-based phase contrast X-ray imaging setup composed of two highly absorbing X-ray gratings, $\mathrm{G}_{\mathbf{0}}$ and $\mathrm{G}_{\mathbf{2}}$, and a phase-shifting X-ray grating, Gı (not to scale).

The fabricated X-ray gratings were tested in a laboratory setup using a Hamamatsu L10101X-ray microsource with a tungsten target and an expected focused source size of 5- $10 \mu \mathrm{m}$. TheX-ray microsource high voltage 
was set to $40 \mathrm{kV}$ and an electron current of $0.200 \mathrm{~mA}$ was employed. The $\mathrm{X}$-ray images were acquired using a scintillator-based sCMOS camera with pixel size of $22 \mu \mathrm{m}$ (1024x1024 pixels) from PhotonicScienceLtd. (UK). The gratings were mounted on motorized stages with nanometer positioning accuracy for alignment and scanning simplicity. The gratings periods $\mathrm{p}_{0}, \mathrm{p}_{1}$ and $\mathrm{p}_{2}$ were chosen to beonemicrometer. Thus, two absorbing gratings made of iridium weremounted in ourlaboratoryX-rayphasecontrastimagingsetup as $\mathrm{G}_{0}$ and $\mathrm{G}_{2}$. The phase-shifting $\mathrm{G}_{1}$ grating was made of silicon and it had a thickness of $25 \mu \mathrm{m}$, thus producing therequired phase shift of $\pi$ rad for an $\mathrm{X}$-ray energy of approximately $20 \mathrm{keV}$.

\subsection{Fabrication of the $X$-ray Gratings}

Phase contrast imaging X-ray gratings require thin silicon supporting substrates to minimize the X-ray absorption when typical photon energies below $30 \mathrm{keV}$ areemployed. Nevertheless, thehandling of very thin substrates can bechallengingbecauseof theirfragilityandfinally, 4-inchsilicon doubleside polished wafers with a thickness of $250 \mu$ m were used to fabricate theX-ray gratings reported here. Theschematics of thefabrication processisdepicted in Fig. 2. Before the lithography step, the silicon wafers were coated with a $100 \mathrm{~nm}$ thick layer of chromium by electron beam evaporation. After that, the grating pattern was produced using conventional UV photolithographyon positive photoresistlayer (MicroChem Corp. S1805) usinga Karl Suss Mask Aligner MA6 in vacuum contact mode. After exposure and development of thephotoresist, thepattern was further transferred into the chromium hard mask by a $\mathrm{RIECl} / \mathrm{CO}_{2}$ based process. Then, the photoresist residuals were removed by immersion in acetone and isopropyl alcohol. Using the patterned 
chromium layer as hard mask, the high aspect ratio silicon structures were produced by a $\mathrm{SF}_{6} / \mathrm{C}_{4} \mathrm{~F}_{8}$ based deep RIE, also commonly know as Bosch process, using an inductively coupled plasma (ICP) Plasmalab 100 system from Oxford Instruments Plasma Technology (UK). The high aspect ratio and high resolution structures were achieved after a careful fine tuning of all process parameters such as thegas flows, chamberpressureand radiofrequency powers. As schematically suggested in Fig. 2(c), the photolithography and deep silicon RIE processes were adjusted to produce a $2 \mu \mathrm{m}$ period silicon grating with a duty cycle of 0.25 . In this way, the one micrometer iridium grating was only produced after a conformal metal coating at both sides of the silicon trenches. Since silicon is a very lowabsorbingmaterial in comparison to iridium, the gaps and the silicon lines of the grating can be regarded as equivalent for theX-ray wavefield that will only be sensitive to effectiveiridium grating period. In thepast, such an approach has been successfully demonstrated for the production of high resolution X-ray diffractive optics[16, 17]. Finally, thelast step in grating fabrication was the conformal deposition of iridium by ALD using iridium acetylacetonate, $\operatorname{Ir}(\mathrm{acac})_{3}$, and oxygen gas, $\mathrm{O}_{2}$, as precursors[13], as depicted in Fig. 2(d). In principle, this deposition method can be used to coat conformally any surface structure by repeatedly supplying two complementary reactant vapors in alternating pulses. Because the chemical reactions are forced to happen entirely on the surface and they are self-limited by the amount of precursor reactant that can be adsorb by the surface, the technique is well-suited for a film growth with almost atomic monolayer accuracy[22, 23]. During the investigations presented here, a Picosun ${ }^{\mathrm{TM}}$ R-200 Advanced ALD tool with the capability 
of delivering a plasma-enhanced oxygen precursor was used. This ALD system has alargereaction chamber suitablefor up to 8-inch wafersandithas 6 separateinlets allowing for several precursors to be supplied. Theinletsare constantly flushed with a flow of nitrogen, even during the ALD cycle steps, to prevent the growth of materials and the likely clogging of the precursor inlet orifices. Asa result, thereactor chamber also requires constant vacuum pumping to prevent an excessive pressure increase, which is kept at around 10 mTorr. Since the precursor species are supplied by mixing the precursor vapor with inlet flow of nitrogen, the constant vacuum pumping of the reactor chamber is artificially limiting the precursor exposure of thesurface beingcoated. However, thesystem can beoperated usinga Picoflow ${ }^{\mathrm{TM}}$ diffusion enhanceroperation modewhich combines thetemporary closureof the vaccuum pumping valve during the precursor delivery pulse of every ALD cycle with the addition of a stainless steel lid to reduce the reactor chamber size. Using this operation mode, the effective dosing time is increasedand thediffusion of theprecursor speciesispromoted. Concerningtheprecursor species, the $\operatorname{Ir}(\mathrm{acac})_{3}$, that is a powder at room temperature, is kept at high temperature (195 $\operatorname{deg}$ C) and low pressure, so that it sublimates inside its reservoir. The precursor is delivered by shortly opening the containervalves and mixing the $\operatorname{Ir}(\mathrm{acac})_{3}$ vapor with nitrogen flow through the line. Therefore, the Ir(acac) ${ }_{3}$ vapor pressure limits the total amount of precursor that can be delivered for a single ALD pulse to the reactor chamber. The $\mathrm{O}_{2}$ precursor can be both delivered as gas for an exclusively thermal reaction or through a plasma generator for a plasma-enhanced ALD process witha typical radiofrequency power of $2000 \mathrm{~W}$. 
(a) Lithography on photoresist

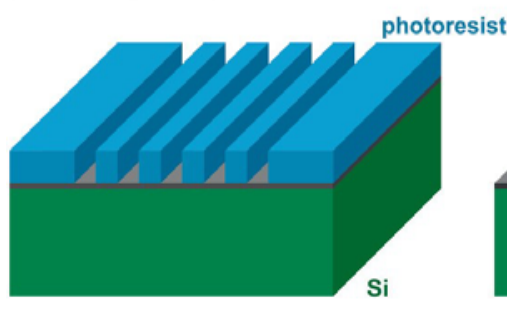

(c) Deep reactive ion etching of $\mathrm{Si}$

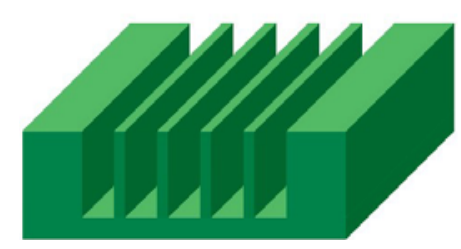

(b) Pattern transfer to $\mathrm{Cr}$ hard mask

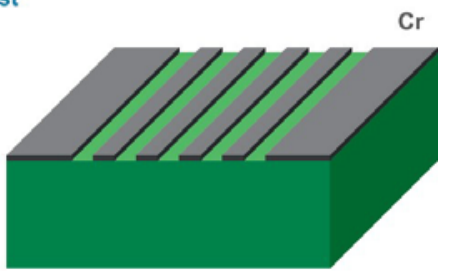

(d) Atomic layer deposition of Ir

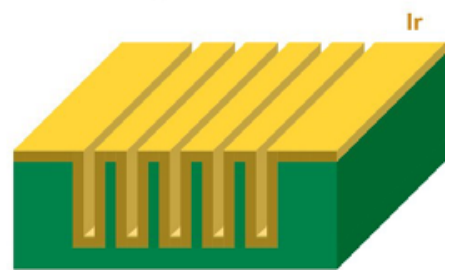

Figure 2: Fabrication steps of the X-ray gratings by combining deep reactive ion etching of silicon and atomic layer deposition of iridium. After the photolithography step (a), the grating pattern is transferred into the chromium hard mask, (b), used during the silicon deep reactive ion etching, (c). The final atomic layer deposition step, (d), coats the silicon trenches with a conformal layer of iridium.

\section{Results and Discussion}

\subsection{Optimization of X-ray Grating Fabrication}

The fabrication procedure of the X-ray gratings reported here relies on combining two well-established techniques - silicon deep RIE and ALD of iridium - thatrequiredanexhaustivetailoringforourparticularpurpose. On the one hand, the optimization of theX-ray grating fabrication started with thefine-tuning of the deep reactiveion etchingbybalancingtheetchingand passivation times to achieve the required depth of $30 \mu \mathrm{m}$, vertical sidewalls 
with a $2 \mu \mathrm{m}$ periodicity and the duty cycle of 0.25 . The details of the deep RIErecipearesummarizedin Tab. 1anditsresultsareshownin thescanning electron micrograph of Fig. 3(a). It can be seen that all the requirements are met and that the targeted $500 \mathrm{~nm}$ silicon lines with a periodicity of $2 \mu \mathrm{m}$ and depth of $30 \mu \mathrm{m}$ have been achieved.

Table 1: Detailed parameters of the silicon deep reactive ion etching recipe. An ICP Plasmalab 100 system from Oxford Instruments Plasma Technology (UK) was used.

\begin{tabular}{lccc}
\hline & Etching Step & Passivation Step \\
\hline Temperature [deg C] & & 0 & \\
Number of Cycles & & 350 & \\
Presurre [mtorr] & & 20 & \\
$\mathrm{SF}_{6}$ Flow [sccm] & 100 & & 5 \\
$\mathrm{C}_{4} \mathrm{~F}_{8}$ Flow [socm] & 5 & 100 \\
ICP Power & 600 & 600 \\
RF Power & 30 & 20 \\
Step Time [s] & 3.0 & 3.0 \\
\hline
\end{tabular}

On the other hand, the optimization of the ALD recipe was both approached from a theoretical point of view and from the experimental considerations directly derived from the operational aspects of thePicosun ${ }^{\mathrm{TM}}$ R-200 Advanced ALD tool reported in Sec. 2.2. From a theoretical standpoint, ALD is expected to coatconformallyanysurfaceif sufficient precursor exposure and dosing times are supplied. Nevertheless, a complete step coveragewillstronglydepend on theparticularaspectratio of thestructuresunder consideration[22, 24]. For example, and from a kinetic theoretical model, it has been shown that a hole with aspect ratio 43 is expected to require a pre- 
cursor exposure of about 3000 times larger than a flatsurface[22]. Therefore, high aspectratio structures will require maximizing the precursor exposure of the surface by increasing the precursor partial pressure (i.e. concentration) in the reactor chamber or at least, by increasing the dosing time to promote theprecursor diffusion and to ensure the precursor saturation of thesurface being coated. Taking this into account, the use of silicon gratings with a periodicity of $2 \mu \mathrm{m}$ and a duty cycle 0.25 is clearly more advantageous than trying to completely fill smaller trenches of $500 \mathrm{~nm}$.

After a fewexperimental trials, it was confirmed that theessential key for a successful ALD conformal coating was to deliver enough $\operatorname{Ir}(\mathrm{acac})_{3}$ precursor and to promote its diffusion into the silicon trenches by increasing the ALD cyclestep timesand byemploying thePicoflow ${ }^{\mathrm{TM}}$ operation modeof thesystem. The complete parameter details of the ALD recipe that delivered the highest conformal coating to the $30 \mu \mathrm{m}$ deep silicon trenches are contained in Tab. 2. This optimized iridium ALD recipestarted with a deposition of a thin $\left(\sim 10 \mathrm{~nm}\right.$ ) layer of $\mathrm{Al}_{2} \mathrm{O}_{3}$ to promote the nucleation of the iridium layer on thesilicon surface. After that, theactual iridium deposition was dividedinto two separate processes: (1) a short deposition using oxygen plasma-enhanced recipe to obtain a uniform nucleation; and (2) a very long deposition using a thermal oxygen process to prioritize the conformality of theiridium coating. It is reported that the use of a plasma precursor is beneficial to obatin a nucleation of smaller grain which will result in a much smoother layer as the deposition progresses. However, it has also been reported that for long depositions the plasma can lead to unconformal deposition as recombination problems can occurs for high aspect ration structures, that is, the precursor 
ion species may react before reaching the bottom region of the trench[23]. Theresults of theALDrecipecanbeobservedin thescanningelectronmicrograph of Fig. 3(b) and they demonstrate the conformal coating of the $30 \mu \mathrm{m}$ deep silicon trenches. Figures 3(c) and (d) compare the iridium thickness obtain at the top and the bottom of the silicon template and Fig. 3(d) shows a larger portion of the fabricated grating.

Table 2: Detailed parameters of the iridium atomic layer deposition recipe for conformal coating of high aspect ratio silicon trenches. The recipe is divided in three separate processes: a deposition of a thin layer of $\mathrm{Al}_{2} \mathrm{O}_{3}$ to ensure the iridium growth; the nucleation of iridium using $\mathrm{O}_{2}$ plasma-based recipe to obtain smoother iridium layer; the deposition of the required iridium thickness using thermal O2-based recipe to prioritize a conformal coating of the silicon.

\begin{tabular}{|c|c|c|c|c|c|c|}
\hline & \multicolumn{2}{|c|}{$\mathbf{A l}_{2} \mathbf{O}_{3}$ Deposition } & \multicolumn{2}{|c|}{ Ir Nucleation } & \multicolumn{2}{|c|}{ Ir Deposition } \\
\hline & 1st ALD Pulse & 2nd ALD Pulse & 1st ALD Pulse & 2nd ALD Pulse & 1st ALD Pulse & 2ndALD Pulse \\
\hline Temperature [deg C] & \multicolumn{2}{|c|}{370} & \multicolumn{2}{|c|}{370} & \multicolumn{2}{|c|}{370} \\
\hline Number of Cycles & \multicolumn{2}{|c|}{300} & \multicolumn{2}{|c|}{800} & \multicolumn{2}{|c|}{11000} \\
\hline Thickness [nm] & \multicolumn{2}{|c|}{10} & \multicolumn{2}{|c|}{30} & \multicolumn{2}{|c|}{480} \\
\hline Presurre [mTorr] & 12 & 12 & 12 & 12 & 20 & 12 \\
\hline Precursor & TMA & $\mathrm{H}_{2} \mathrm{O}$ & $\operatorname{Ir}(\mathrm{acac})_{3}$ & $\mathrm{O}_{2}$ plasma & $\operatorname{Ir}(\mathrm{acac})_{3}$ & $\mathrm{O}_{2}$ gas \\
\hline Line Gas Flow [sccm] & 150.0 & 150.0 & 150.0 & 60 & 150.0 & 100.0 \\
\hline Pulse Time [s] & 0.5 & 0.5 & 1.0 & 6.0 & 4.0 & 10.0 \\
\hline Purge Time [s] & 6.0 & 6.0 & 6.0 & 6.0 & 35.0 & 35.0 \\
\hline Picoflow ${ }^{\mathrm{TM}}$ Operation Mode & No & No & No & No & Yes, $25.0 \mathrm{~s}$ & Yes, $25.0 \mathrm{~s}$ \\
\hline Total Cycle Time [s] & \multicolumn{2}{|c|}{13} & \multicolumn{2}{|c|}{19} & \multicolumn{2}{|c|}{120} \\
\hline Total Deposition Time & \multicolumn{2}{|c|}{$<1$ hour } & \multicolumn{2}{|c|}{$\sim 4$ hours } & \multicolumn{2}{|c|}{$\sim 15$ days } \\
\hline
\end{tabular}

The current ALD recipe using the Picosun ${ }^{\mathrm{TM}} \mathrm{R}-200$ Advanced ALD tool was able to deliver a conformal coating of iridium for 3 grating substrate pieces of $2 \times 2 \mathrm{~cm}^{2}$ with a singleALD run. However during the experimental trials, it wasfound that theiridium deposition wasnot completely conformal at the central regions of a full 4-inch silicon wafer with a $\sim 7 \times 7 \mathrm{~cm}^{2}$ grating patterned area. In this latter case, it is suspected that the total amount of 
$\operatorname{Ir}(\mathrm{acac})_{3}$ precursor supplied to the chamber was not sufficient to saturate thelarger area of the fully patterned 4-inch silicon wafer. This result clearly points theimportance of operational aspects of theparticularALD systemin use. It is expected that with a dedicated ALD system that would be ableto deliver the $\operatorname{Ir}(\mathrm{acac})_{3}$ precursorfrommorethan onereservoirat thesametime wouldallowthecoatingoflargergratingareasanditwould probablydecrease substantially thevery longALD pulsetimes required by theequipment used for investigations reported here.

\subsection{Performance of the $X$-ray Gratings}

The X-ray absorbing iridium gratings were characterized at our laboratory X-ray phase imaging setup. A picture of the actual system is shown in Fig. 4(a). The setup was arranged to employ the 9th and 13th Talbot order configurations with a gratingseparation distances of 3.7 and $5.2 \mathrm{~cm}$, respectively. The fabricated gratings were mounted and aligned using the motorized positioning systems. During the tests, a sample consisting of polystyrene microspheres with a diameter of $700 \mu \mathrm{m}$ was used. The differential phase contrast images of the spheres at the 9th and 13th Talbot order configurations are respectively shown in Fig. 4(b) and (c). Comparing the two images, the highest sensitivity is achieved by the 13th Talbot order as it is theoretically expected[21]. Themain advantageof havingsmallerperiodsisthereduction of the grating separation distance to obtain a similar sensitivity. For example, when using $3 \mu$ mgratingperiodsaseparation distancebetween gratings of $16.3 \mathrm{~cm}$ would be required for a similar sensitivity to the one achieved by setup reported here. In addition, using shorter grating separation distances is very beneficial in terms of photon flux when using laboratory X-ray mi- 
crosources. The measured fringe grating visibility, that is an evaluation of the performance of the X-ray phase imaging setup, was found to be $11.5 \%$ and $12.1 \%$ for the 9 th and 13th Talbot orders. The visibility histograms are shown in the inset plots of Fig. 4(b) and (c). Finally, it can be observed that the phasecontrast images are clean of defects, which in turn is an indication of the excellent quality of the fabricated one micrometer period gratings.

\section{Conclusions}

In summary, wehavedemonstrated the fabrication and characterization of high aspect ratio iridium gratings with a period of one micron and a depth of $30 \mu \mathrm{m}$ for X-ray phase contrast imaging. The combination of deep RIE of silicon and atomiclayer deposition of iridium has been proven as an effective fabrication method to produce high quality X-ray absorbing gratings. The technique has the potential of producing even smaller period gratings over

larger areas ( $>5 \times 5 \mathrm{~cm}^{2}$ ) if used together with new emerging lithography technologies such as Displacement TalbotLithography[25] that havethecapability of producing sub-micrometer grating patterns over large areas ( 4 and 8-inch wafers). Webelieve that theproposed method can beused to provide high quality gratings that will greatly enhance the sensitivity of gratingbased X-ray phase contrast imaging and broaden its range of applications. In addition, the technique could be easily transferred for the production of other microcomponents and optical devices. 


\section{Acknowledgements}

The authors would like to thank Dr. Z. Wang and Dr. C. Arboleda from thePaul ScherrerInstitut (Switzerland) for assistanceduring thephasecontrast X-ray imaging experiments. This work has been partially funded by theERC-2012-SRG310005-PhaseX grantand by theSwiss National Science Foundation, SNSF Grant Number 159263.

\section{References}

[1] A. Momose, S. Kawamoto, I. Kowama, Y. Hamaishi, K. Takai, Y. Suzuki, Demonstration of x-ray talbot interferometry, J pn. J . Appl. Phys. 42 (2003) L866- L868.

[2] T. Weitkamp, A. Diaz, C. David, F. Pfeiffer, M. Stampanoni, P. Cloetens, E. Ziegler, X-ray phase imaging with a grating interferometer, Opt. Express 13 (2005) 2062- 2068.

[3] F. Pfeiffer, M. Bech, O. Bunk, P. Kraft, E. F. Eikenberry, C. G. C. Brnnimann, C. David, Hard-x-ray dark-field imagingusinga gratinginterferometer, Nat. Materials 7 (2008) 134- 137.

[4] T. Weitkamp, B. Nhammer, A. Diaz, C. David, X-ray wavefront analysis and optics characterization with a grating interferometer, Appl. Phys. Lett. 86 (2005) 054101.

[5] S. Rutishauser, L. Samoylova, J . Krzywinski, O. Bunk, J . Grnert, H. Sinn, M. Cammarata, D. M. Fritz, C. David, Exploring the wave- 
front of hardx-ray free-electron laserradiation, Nat.Commun.3(2012) 947.

[6] Z. Wang, N. Hauser, G. Singer, M. Trippel, R. Kubik-Huch, C. Schneider, M. Stampanoni, Non-invasiveclassification of microcalcifications with phase-contrastx-raymammography, Nat. Commun. 5(2014) 1-9.

[7] C. Arboleda, Z. Wang, T. Koehler, G. Martens, U. V. Stevendaal, M. Bartels, P. Villanueva-Perez, E. Roessl, M. Stampanoni, Sensitivitybased optimization for the design of agratinginterferometer forclinical x-rayphasecontrast mammography, Opt. Express 25(6) (2017) 6349_ 6364.

[8] L. B. Gromann, F. De Marco, K. Willer, P. B. Noël, K. Scherer, B. Renger, B. Gleich, K. Achterhold, A. A. Fingerle, D. Muenzel, S. Auweter, K. Hellbach, M. Reiser, A. Baehr, M. Dmochewitz, T. J. Schroeter, F. J. Koch, P. Meyer, D. Kunka, J . Mohr, A. Yaroshenko, H.-I. Maack, T. Pralow, H. van der Heijden, R. Proksa, T. Koehler, N. Wieberneit, K. Rindt, E. J. Rummeny, F. Pfeiffer, J. Herzen, Invivo X-ray Dark-Field Chest Radiography of a Pig, Scientific Reports 7 (2017) 4807.

[9] H. Wen, A. A. Gomella, A. Patel, S. K. Lynch, N. Y. Morgan, S. A. Anderson, E. E. Bennett, X. Xiao, C. Liu, D. E. Wolfe, Subnanoradian Xray phase-contrast imaging using a far-field interferometer of nanometric phase gratings, Nat. Commun. 4 (2013) 2659.

[10] C. David, J. Bruder, T. Rohbeck, C. Grunzweig, C. Kottler, A. Diaz, 
O. Bunk, F. Pfeiffer, Fabrication of diffraction gratings for hardx-ray phase contrast imaging, Microelectron. Eng. 84 (2007) 1172- 1177.

[11] D. Noda, A. Tokuoka, T. Hattori, Fabrication of high aspect ratio x-ray grating using silicon dry etching method, AIP Conf. Proc. 1466 (2012) 187.

[12] J. Mohr, T. Grund, D. Kunka, J. Kenntner, J. Leuthold, J. Meiser, J . Schulz, M. Walter, High aspectratiogratings for x-ray phasecontrast imaging, AIP Conf. Proc. 1466 (2012) 41- 50.

[13] T. Aaltonen, M. Ritala, V. Sammelselg, M. Leskela, Atomic layer deposition of iridium thin films, J . Electrochem. Soc. 151(8) (2004) G489G492.

[14] E. Shkondin, O. Takayama, J . M. Lindhard, P. Voss Larsen, M. Dysseholm Mar, F.J ensen, A. V. Lavrinenko, Fabrication of high aspect ratio tio $_{2}$ and $\mathrm{al}_{2} \mathrm{O}_{3}$ nanogratings by atomiclayerdeposition, J . Vac. Sci. Technol. A 34(3) (2016) 031605.

[15] R. Zazpe, M. Knaut, H. Sopha, L. Hromadko, M. Albert, J. Prikryl, V. Grtnerov, J. W. Bartha, J. M. Macak, Atomic layer deposition for coating of high aspect ratio tion nanotube layers, Langmuir 32(41) (2016) 10551- 10558.

[16] K. J efimovs, J. Vila-Comamala, T. Pilvi, J . Raabe, M. Ritala, C. David, Zone-doubling technique to produce ultrahigh-resolution x-ray optics, Phys. Rev. Lett. 99 (2007) 264801. 
[17] J. Vila-Comamala, S. Gorelick, V. A. Guzenko, E. Farm, M. Ritala, C. David, Dense high aspect ratio hydrogen silsesquioxane nanostructures by 100 kev electron beam lithography, Nanotechnology 21(2010) 285305.

[18] D.Attwood, SoftX-raysandExtremeUltravioletRadiation, Cambridge University Press, Cambridge, UK, 2000.

[19] F. Pfeiffer, O. Bunk, C. David, M. Bech, G. L. Duc, A. Bravin, P. Cloetens, High-resolution brain tumor visualization using threedimensional x-ray phase contrast tomography, Phys. Med. Biol. 52 (2007) 6923-6930.

[20] M.-C. Zdora, J . Vila-Comamala, G. Schulz, A. Khimchenko, A. Hipp, A. C. Cook, D. Dilg, C. David, C. Grünzweig, C. Rau, P. Thibault, I.Zanette, X-ray phasemicrotomographywith asinglegratingfor highthroughput investigations of biological tissue, Biomed. Opt. Express 8(2) (2017) 1257- 1270.

[21] T. Donath, M. Chabior, F. Pfeiffer, O. Bunk, E. Reznikova, J . Mohr, E. Hempel, S. Popescu, M. Hoheisel, M. Schuster, B.J oachim, C. David, Inverse geometry for grating-based $\mathrm{x}$-ray phase-contrast imaging, $\mathrm{J}$. Appl. Phys. 106 (2009) 054703.

[22] R. G. Gordon, D. Hausmann, E. Kim, J. Shepard, A kinetic model for step coverage by atomic layer depsotion in narrow holes or trenches, Chem. Vap. Depostion 9 (2) (2003) 73- 78. 
[23] H. C. M. Knoops, E. Langereis, M. C. M. van der Sanden, W. M. M. Kessels, Confromality of plasma-assisted ald: Physical processes and modeling, J . Electrochem. Soc. 157 (12) (2010) G241- G249.

[24] A. Yanguas-Gil, J . W. Elam, Self-limited reaction-diffusion in nanostructured substrates: Surface coveragedynamics and analytic approximations to ald saturation times, Chem. Vap. Deposition 18 (2012) 46- 52.

[25] H. H. Solak, C. Dais, F. Clube, Displacement talbot lithography: a newmethod for high-resolution patterning of largeareas, Opt. Express 19(11) (2011) 10686- 10691. 

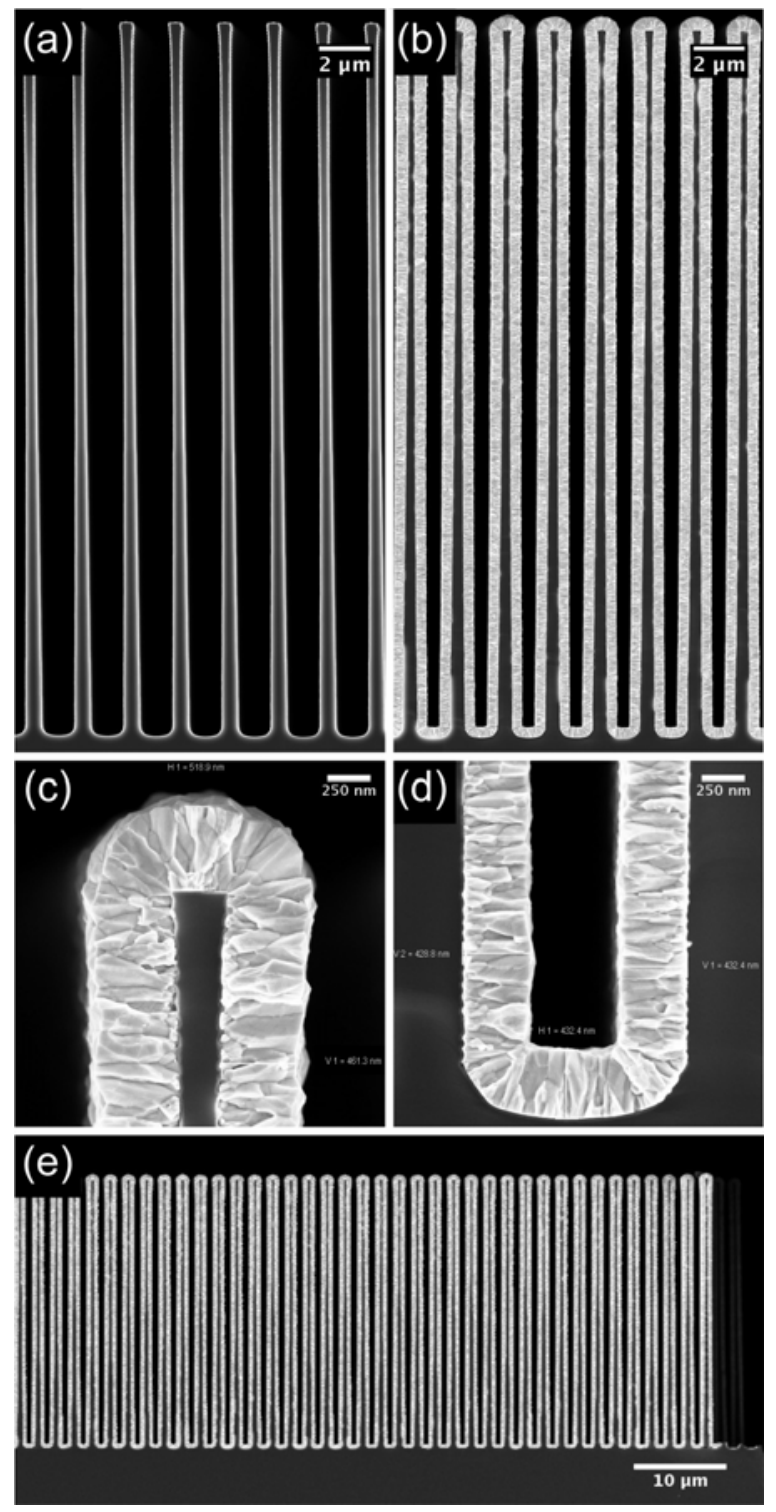

Figure 3: (a) Scanning electron micrograph of the silicon grating fabricated by deep reactive ion etching with a periodicity of $2 \mu \mathrm{m}$, a depth of $30 \mu \mathrm{m}$ and a duty cycle of 0.25. (b) One micrometer iridium grating after the atomic layer deposition process. (c) and (d) demonstrate the obtained conformality by comparing the thickness of iridium at the top and bottom of the silicon trenches. (e) (a) Low magnification scanning electron micrograph of the one micrometer iridium X-ray grating. 

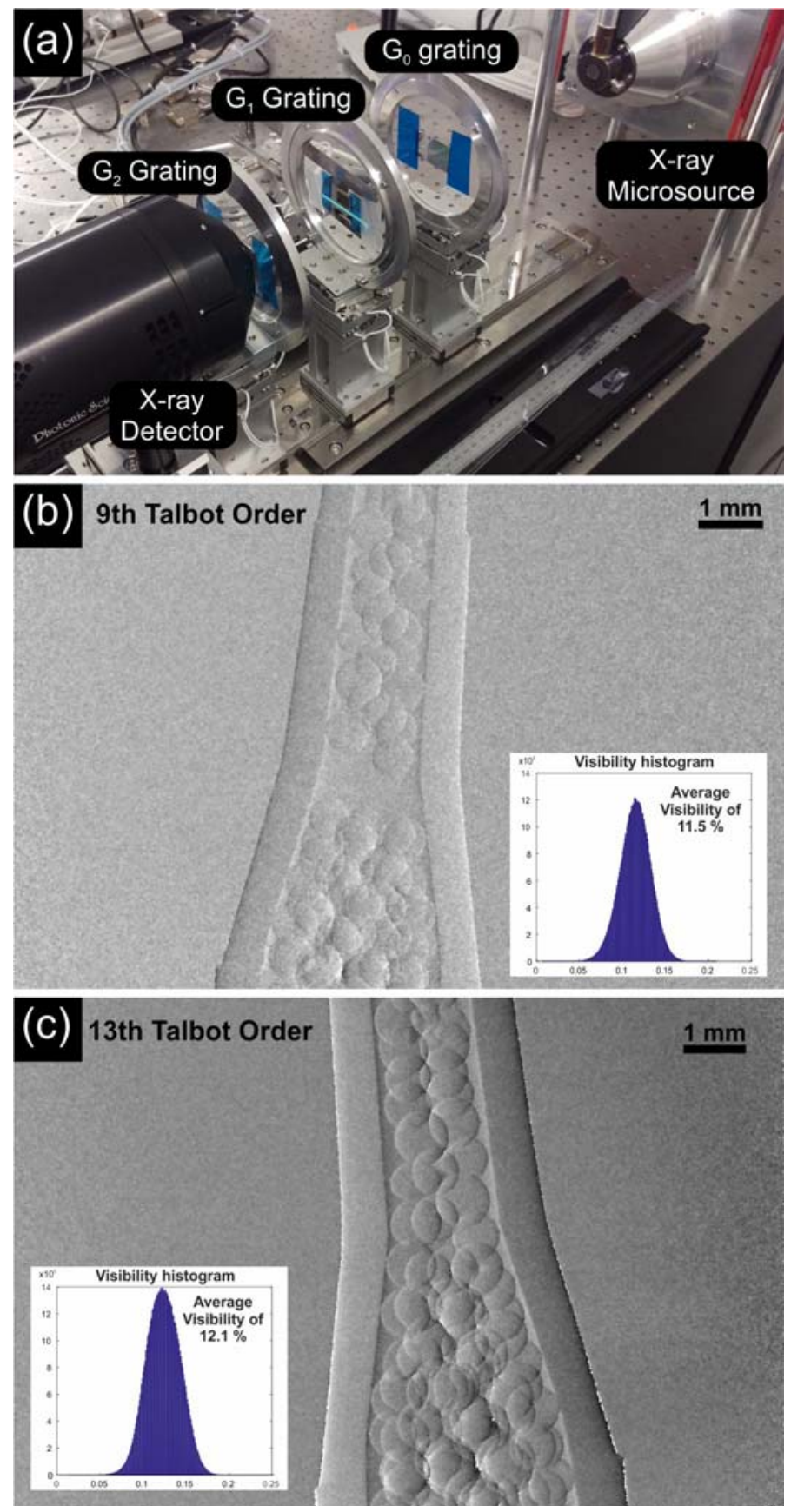

Figure 4: (a) Picture of the laboratory X-ray phase imaging setup with the fabricated one micrometer period iridium gratings mounted as Go and G2. (b) and (c) display the differential phase contrast images of a sample made of $700 \mu \mathrm{m}$ silicon oxide spheres

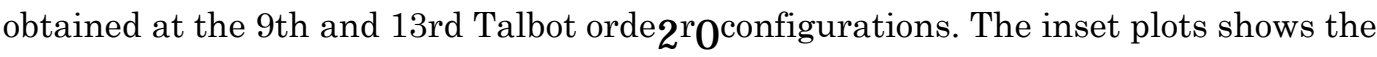
histogram of the visibility obtained, that is a direct evaluation parameter of the gratingbased X-ray phase contrast imaging setup. 